\title{
Estimation of the Genetic Parameters of the Growth Characteristics in Rice Crop (Oryza sativa L.) by Effect of two Methods Irrigation
}

\author{
Abdullah F. Serheed ${ }^{a}$ \\ Haider B. Ahmed ${ }^{\text {b }}$ \\ Technical college AL-Musaib \\ abdfade168@yahoo.com \\ Submission date:- 19/8/2018 Acceptance date:- 23/9/2018 \\ Publication date:- 22/1/2019 \\ Keywords: Heritability, genotype, variance, irrigation.

\begin{abstract}
Field experiments were carried out for 2017 agricultural season in Babylon / Musaib - Albojasem region $35 \mathrm{~km}$ west north of the governorate to evaluate the performance of seven genotypes of rice (Oryza sativa L.) Genetic, environmental and phenotypic variances, heritability percent in the broad sense, genetic and phenotypic Different Coefficients, effect of irrigation methods (flooding and intermittent irrigation) of the genotypes (Amber33, Dijla, Mashkhab 2, Forat, Pernameg4, Yasmin and Ghadir) The research center of the rice in Al-Mashkhab using the experiment of split plots in randomized complete Block design (RCBD) with three replicates. The results can be summarized as follows:

1.The genotypes showed significant differences on the $5 \%$ probability level for all studied traits.

2. The genotype Amber 33 superior to all other traits except for the number of effective branches for genotype Forat.

3. The method of irrigation by flooding showed significant superiority of all studied traits.

4. The genetic variance values were higher than the environmental variability values of all traits except for the number of branches.

5.The estimates of heritability values in the broad sense indicated that they were high for all traits .

6.The values of the phenotypic and genetic differences were different between low values of the number of days from planting to 50\% flowering and medium for the other traits except for the area of the leaf it was high for the irrigation methods and the low of the number of branches. panicle.
\end{abstract}




\section{تقدير المعالم الور اثية لصفات النمو لمحصول الرز (Oryza sativa L.) بتأثير طريقتين للري}

حيلر باسم احمد

عبدالله فاضل سرهيد

الكلبة الثقنية، المسبب

abdfadel68@yahoo.com

الخلاصة

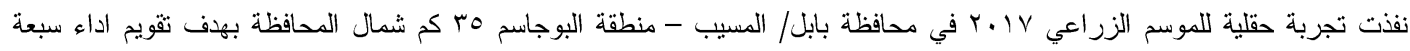

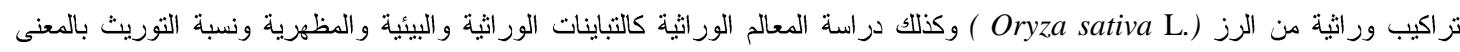

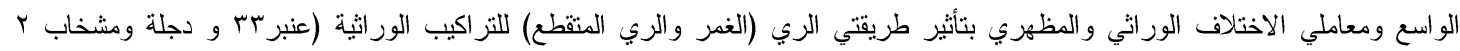
وفرات وبرنامج؛ وياسمين و غدير) والتي تم الحصول عليها من محطة ابحاث الرز في المشخاب باستعمال تجربة الالواح المنشقة بتصميم

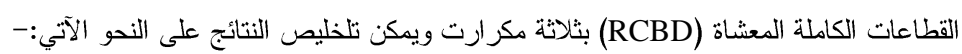
1. اختلفت التز اكيب الور اثية معنويا على مستوى احتمال ه\% لجميع الصفات المدروسة. r. تفوق التركيب الور اثي عنبر بr في جميع الصفات المدروسة باستثناء صفة عدد التفرعات الفعالة التي تفوق فيها التركيب الور اثي فرات. r. تقوقت طريقة الري بالغمر معنوياً لجميع الصفات المدروسة. عـ. كانت قيم التباين الور اثي لما يشكله من قيمة التباين الكلي عالية لجميع الصفات باستثناء صفة عدد الافرع .دالية للموقع الاول فقط. ه. اشارت تقديرات قيم التوريث بالمعنى الواسع الى انها كانت عالية لجميع الصفات. جا. كانت قيم معاملي الاختلاف المظهري و الور اثي مختلفة بين واطئة لصفات عدد الايام من الزر اعة حتى .0\% تزهير ومتوسطة لبقية الصفات

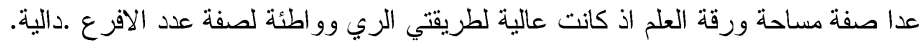
الكلمات الدالة: توريث، تز اكيب ور انثة، تباينات، ري.

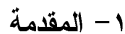

يعدُ محصول الرز (Oryza sativa L) احد محاصيل الحبوب المهمة في العراق و يأتي بالدرجة الثانية بعد الحنطة من حيث الاهمية

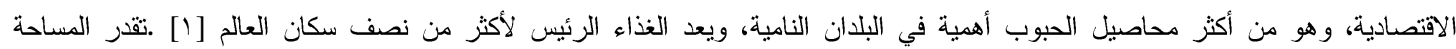

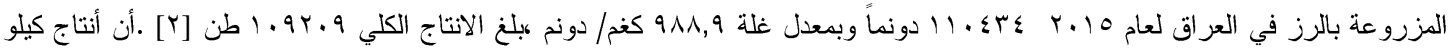

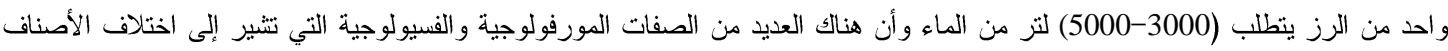
في ميكانيكية تحمل الجفاف [ץ] وفي دراسة حقلية نفذت في محطة أبحاث الرز في المشخاب في محافظة النجف وجد أن أن الاحتياجات المائية للرز

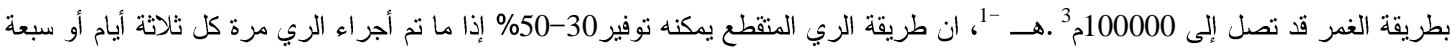

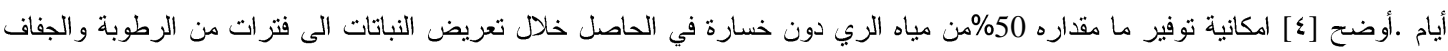

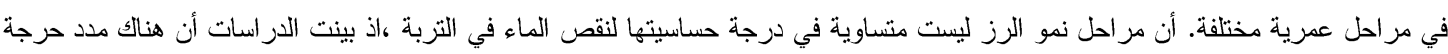

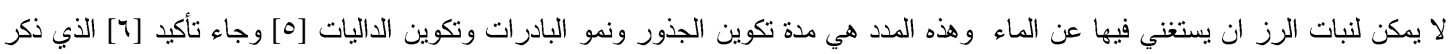

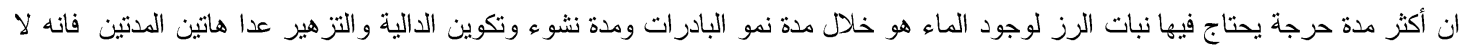

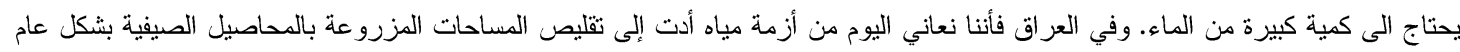

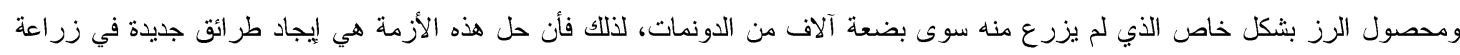

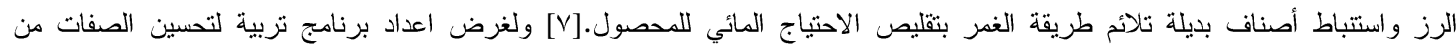

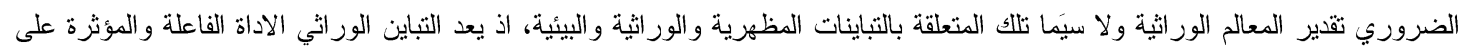

كفاءة الانتخاب. [^]. 
تهدف هذه الدراسة الى:-

ا. ثقييم اداء الاصناف المستعملة في البحث من الرز تحت تاثير طريقتي الري لتحديد افضل الاصناف وطريقة الري. r. تقدير بعض المعالم الور اثثة مثل التباينات المظهرية و الور اثية والبيئية ونسبة التوريث لصفات التر اكيب الور اثثية والتي يتم انتخابها على اساس ادائها الجيد.

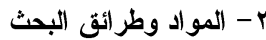

نفذت تجربة حقلية في منطقة المسيب/ ابو الجاسم - محافطة بابل للموسم الزراعي 2017 باستعمال تجربة الألواح المنشقة وفـق تصــميم

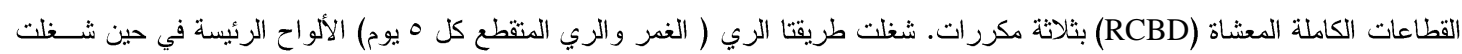

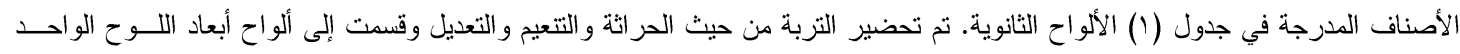
$.^{r} r \times Y$

جدول (1) التر اكيب الوراثية المستعملة في البحث

\begin{tabular}{|c|c|c|}
\hline المنشأ & الصنف & $ث$ \\
\hline محلي & عنبر rr & 1 \\
\hline$=$ & لدجلة & r \\
\hline$=$ & مشخاب r r & $r$ \\
\hline$=$ & فرات & $\varepsilon$ \\
\hline$=$ & برنامج ع & ○ \\
\hline$=$ & ياسمين & 7 \\
\hline$=$ & غدير & $v$ \\
\hline
\end{tabular}

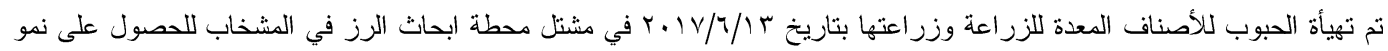
أفضل للشتلات. كانت مساحة المشتل (5×5) م ويكون السقي للمشتل يوميا مع بزل الماء وبقاء المشتل رطب للمساعدة على نمو جذور الشتلات

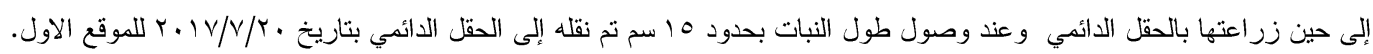

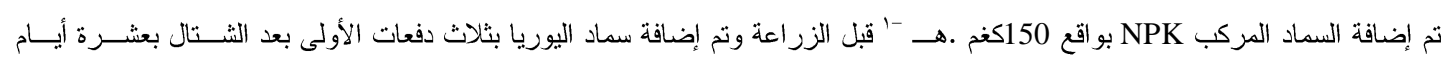

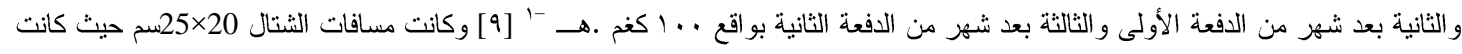

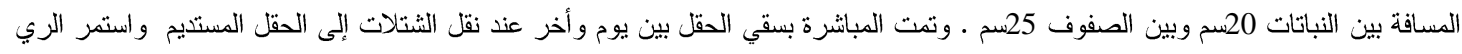

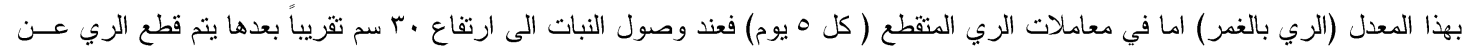

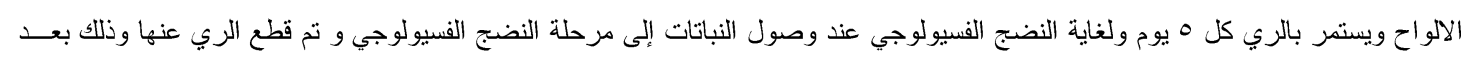

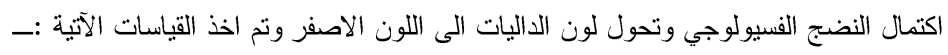

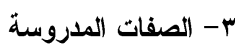

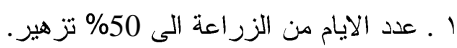

r ب عدد الايام من الزراعة الى النضج الفسيولوجي.

r ـ ارتفاع النبات ( سم) : قيس لعشر نباتات عشو ائية عند الحصاد ويحسب من سطح التربة ولغاية حامل الدالية الرئيس. ع . طول الدالية (سم) : قيس لعشر داليات عشوائية عند الحصاد. ه .عدد الافرع للالية : قيس لعشر داليات عشو ائية عند الحصاد.

كَ. مساحة ورقة العلم ( سمج): قيس لعشر اوراق علم عشوائية, حسب المعادلة الاتية: مساحة ورقة العلم (سم²) = طول الورقة ×عرضها (مسن اعرض منطقة) × 0.74 [1. 


$$
\begin{aligned}
& \text { V. وزن ورقة العلم (ملغم) : وزنت عشر اوراق علم عشوائية بالميزان الكهربائي الحساس. } \\
& \text { ^. النسبة المئوية لعدم الخصب: حسبت على وفق المعادلة الاتية: } \\
& \text { النسبة المئوية لعدم الخصب = (عدد الحبوب الفارغة / عدد الحبوب الكلي) ×100 } \\
& \text { 9. عدد التفر عات الفعالة ( عدد الداليات / م²): حسبت لــ 1م طول ( 0.20م²) عند الحصاد. } \\
& \text { ع - التحليل الإحصائي:- }
\end{aligned}
$$

حللت البيانات إحصائياً بطريقة تحليل التباين , و إستخدام أقل فرق معنوي على مستوى احتمال (0\%) لتشخيص الفروق الاحصـائية بـين المتوسطات الحسابية للمعاملات [1 11] • باستخدام برنامج التحليل الإحصائي Genstat لتحديد مصادر التباين بين المعاملات المدروسة.

- هenetic Analysis التحليل الور اثي

التباينات المظهرية والوراثية والبيئية Phenotypic, Genotypic and Environmental Variances تم تقدير تحليل التباين المظهري

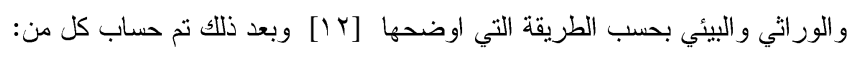

$$
\begin{aligned}
& \sigma_{G}{ }^{2}=\frac{M s g-M s e}{r} \\
& \sigma_{E}{ }^{2}=M s e \\
& \sigma_{P}{ }^{2}=\sigma_{G}{ }^{2}+\sigma_{E}{ }^{2}
\end{aligned}
$$

$$
h_{B . S}^{2}=\frac{\sigma_{G}^{2}}{\sigma_{P}^{2}} x 100
$$

$$
\begin{aligned}
& \text { يمثل التوريث بالمعنى الواسع } H_{B . S}^{2} \\
& \sigma_{G}{ }^{2} \\
& \sigma_{P}{ }^{2}
\end{aligned}
$$

تقديـر قيـــم معاملات الاختلاف المظهري والور اثي مhenotypic and Genotypic Different Coefficients

$$
\text { تم حساب قيم معاملات الاختلاف المظهري و الور اثي حسب الطريقة التي اوضحها [ـ 1] كما ياتي: }
$$

P.C.V $\%=\frac{\sqrt{\sigma_{P}}}{X^{-}} \times 100$

G.C.V $\%=\frac{\sqrt{\sigma_{G}}}{X^{-}} \times 100$ 
يوضح الجدول(ץ) وجود فروق معنوية لصفة عدد الأيام من الزراعة إلى .0\% تز هير اذ تفوق التركيب الور اثي عنبر بr باعطائه اقل عدد

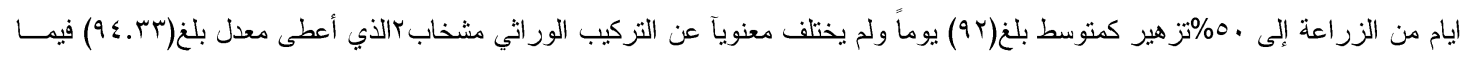

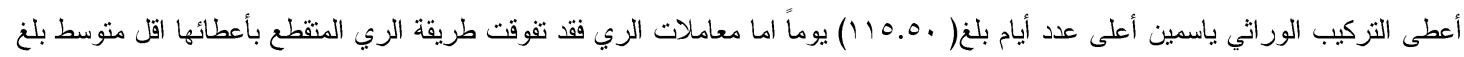

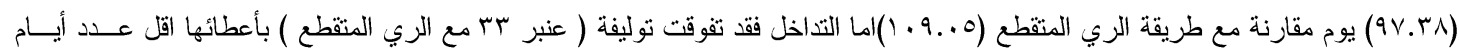

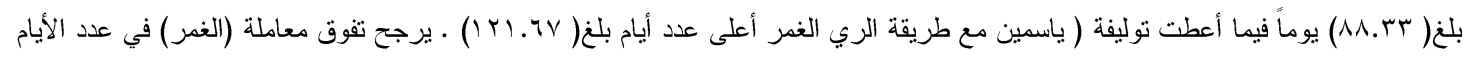

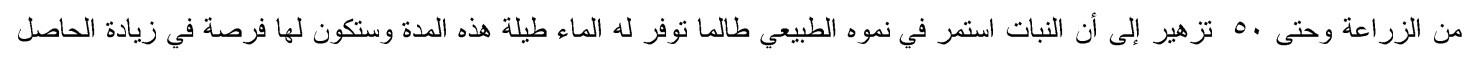
أما المعاملات (الري المتقطع) فأن النباتات حاولت تقصير من مدة نمو ها بسبب عدم الحصول على على كفايتها من الماء.

\section{ب ا.Y - عدد الأيام من الزراعة الى النضج الفسيولوجي}

يوضح الجدول(ץ) وجود فروق معنوية لصفة عدد الأيام من الزراعة إلى النضج الفسيولوجي اذ تفوق التزكيب الوراثي عنبر سب باعطائه

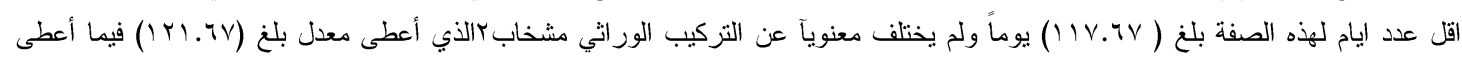

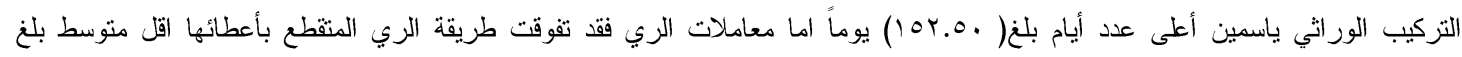

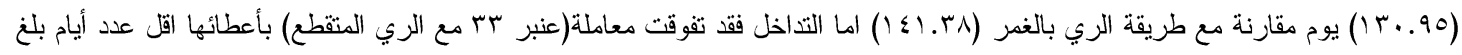

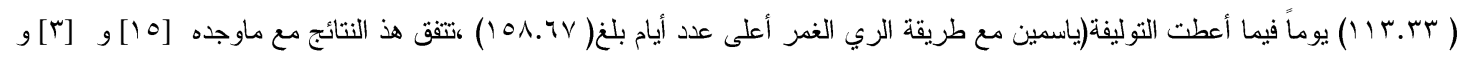


جدول (ץ) قيم المتوسطات الحسابية لصفة عدد الأيام من الزراعة الى ـ ه\% تزهير

\begin{tabular}{|c|c|c|c|}
\hline متوسط التر اكيب & منقطع & غمر & 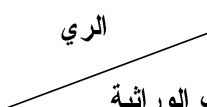 \\
\hline 92.00 & 88.33 & 95.67 & عنبر rr \\
\hline 98.50 & 92.33 & 104.67 & لدجلة \\
\hline 94.33 & 89.67 & 99.00 & مشخاب r \\
\hline 102.83 & 96.33 & 109.33 & فرات \\
\hline 106.17 & 99.00 & 113.33 & برنامج ؛ \\
\hline 115.50 & 109.33 & 121.67 & ياسمين \\
\hline \multirow[t]{2}{*}{ 113.17 } & 106.67 & 119.67 & غدير \\
\hline & 97.38 & 109.05 & متوسط طرق الري \\
\hline للتداخل 5.95 & للري 1.14 & للتر اكيب 4.20 & \%5 $\quad$ L.SD \\
\hline
\end{tabular}

جدول بقيم المتوسطات الحسابية لصفة عدد الأيام من الزراعة الى النضج الفسيولوجي

\begin{tabular}{|c|c|c|c|}
\hline متوسط التراكيب الور اثية & متقطع & غمر & الري \\
\hline 117.67 & 113.33 & 122 & عنبر rr \\
\hline 136 & 131.67 & 140.33 & ل دجلة \\
\hline 121.67 & 116.67 & 126.67 & مشخاب r r \\
\hline 136 & 130.33 & 141.67 & فرات \\
\hline 142.5 & 138.67 & 146.33 & برنامج ؛ \\
\hline 152.5 & 146.33 & 158.67 & ياسمين \\
\hline \multirow[t]{2}{*}{146.83} & 139.67 & 154.00 & غدير \\
\hline & 130.95 & 141.38 & متوسط طرق الري \\
\hline للتداخل 7.22 & للري 3.60 & للتر اكيب 5.11 & $\%$ L.SD \\
\hline
\end{tabular}

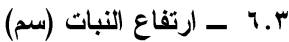

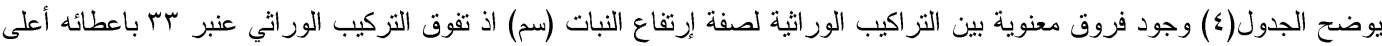

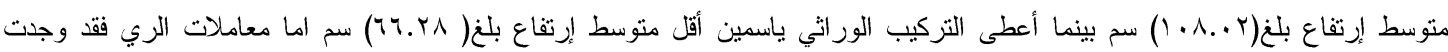

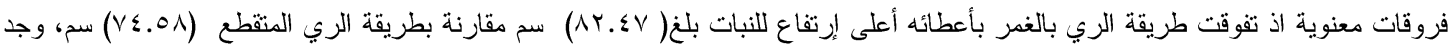

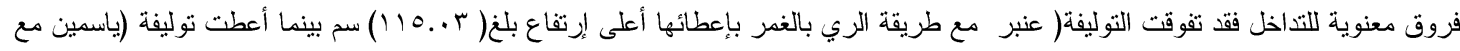


الري المتقطع) أقل متوسط إرتفاع بلغ (r.VV.T) بم يعود السبب في زيادة ارتفاع النبات إلى أن نباتات الرز في معاملة الري بالغمر أخذت حاجتها من مياه الري و لا سيما في المر احل الحساسة من عمر النبات (الإنبات والتز هير) وبحسب طبيعة نمو النبات مقارنة بمعاملة الري المتقطع.

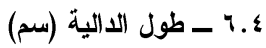

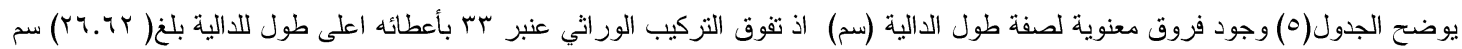

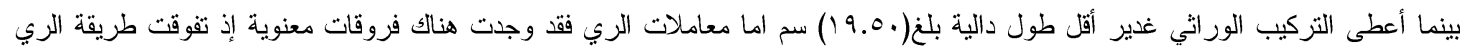

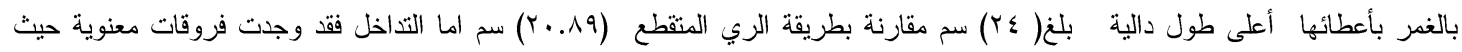

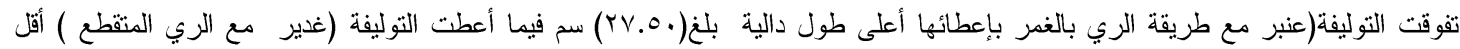

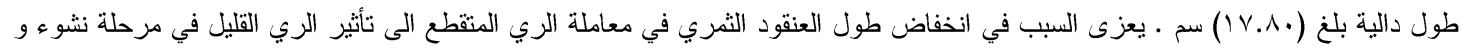

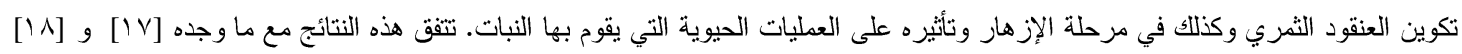

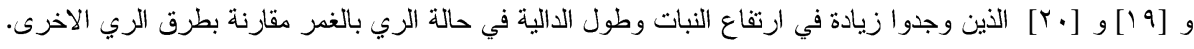

جدول (§) قيم المتوسطات الحسابية لصفة|رتفاع النبات (سم)

\begin{tabular}{|c|c|c|c|}
\hline متوسط التراكيب الور اثية & متقطع & غمر & 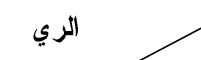 \\
\hline 108.02 & 101.00 & 115.03 & عنبر rr \\
\hline 77.62 & 74.13 & 81.10 & دجلة \\
\hline 73.03 & 70.47 & 75.6 & مشخاب r \\
\hline 77.48 & 72.80 & 82.17 & فرات \\
\hline 74.62 & 70.43 & 78.8 & برنامج ؛ \\
\hline 66.28 & 62.77 & 69.8 & ياسمين \\
\hline \multirow[t]{2}{*}{72.62} & 70.47 & 74.77 & غدير \\
\hline & 74.58 & 82.47 & متوسط طرق الري \\
\hline للتداخل 3.06 & للري 4.89 & للتر اكيب 2.16 & \%० L.SD \\
\hline
\end{tabular}

جدول (•) قيم المتوسطات الحسابية لصفة طول الدالية (سم)

\begin{tabular}{|c|c|c|c|}
\hline متوسط التر اكيب الور اثية & متقطع & غمر & 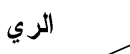 \\
\hline 26.62 & 25.73 & 27.5 & عنبر rr \\
\hline 21.48 & 20.60 & 22.37 & دجلة \\
\hline 24.05 & 22.2 & 25.9 & مشخاب r r \\
\hline 21.3 & 19.2 & 23.4 & فرات \\
\hline 21.45 & 19.8 & 23.1 & برنامج ؛ \\
\hline 22.68 & 20.87 & 24.5 & ياسمين \\
\hline 19.50 & 17.80 & 21.2 & غدير \\
\hline
\end{tabular}




\begin{tabular}{|c|c|c|c|}
\hline & 20.89 & 24 & متوسط طرق الري \\
\hline للتداخل 1.58 & للري 0.24 & للتر اكيب 1.12 & $\%$ L.SD \\
\hline
\end{tabular}

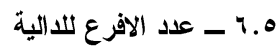

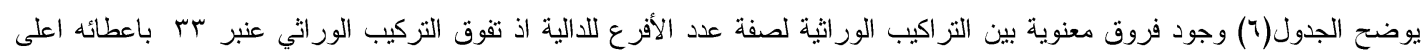

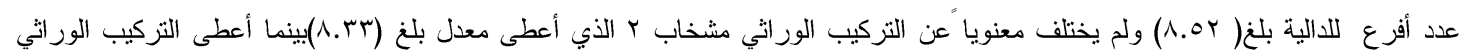

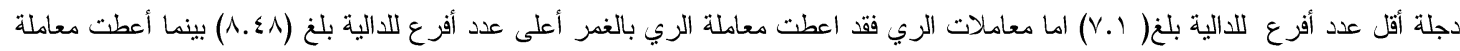

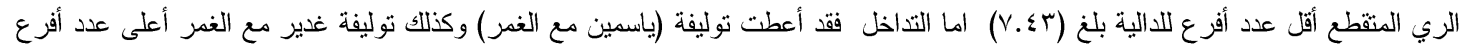

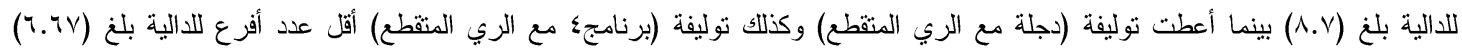

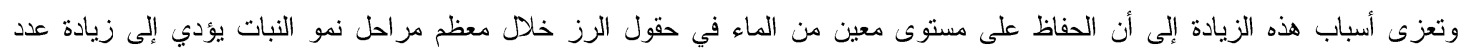
الأفرع/ دالية.

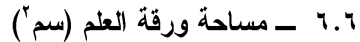

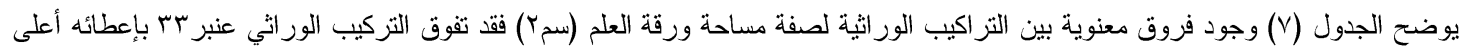

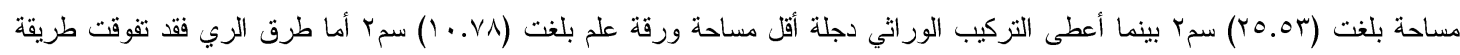

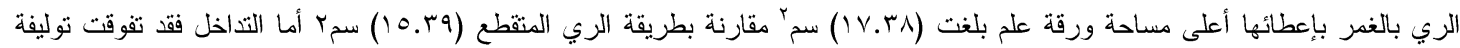

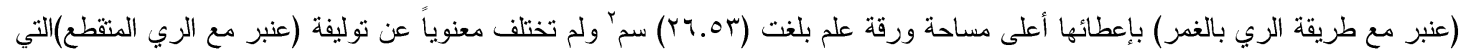

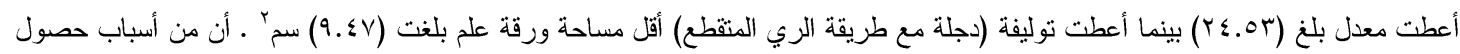

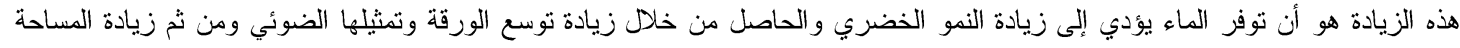

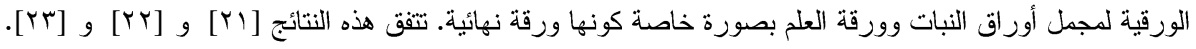

جدول (ا) قيم المتوسطات الحسابية لصفة عدد الافرع للالية

\begin{tabular}{|c|c|c|c|}
\hline متوسط التر اكيب & متقطع & غمر & 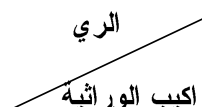 \\
\hline 8.52 & 8.4 & 8.63 & عنبر rr \\
\hline 7.1 & 6.67 & 7.53 & لدجلة \\
\hline 8.33 & 8.17 & 8.5 & مشخاب r \\
\hline 7.97 & 7.27 & 8.67 & فرات \\
\hline 7.63 & 6.67 & 8.6 & برنامج ؛ \\
\hline 8.20 & 7.70 & 8.7 & ياسمين \\
\hline \multirow[t]{2}{*}{7.92} & 7.13 & 8.7 & غدير \\
\hline & 7.43 & 8.48 & متوسط طرق الري \\
\hline للتداخل 0.46 & للري 0.40 & للتر اكيب 0.33 & \%॰ L.SD \\
\hline
\end{tabular}


جدول (V) قيم المتوسطات الحسابية لصفة مساحة ورقة العلم (سمץ)

\begin{tabular}{|c|c|c|c|}
\hline متوسط التر اكيب الور اثية & متقطع & غمر & الري ل \\
\hline 25.53 & 24.53 & 26.53 & عنبر rr \\
\hline 10.78 & 9.47 & 12.1 & لدجلة \\
\hline 15.53 & 14.73 & 16.33 & مشخاب r r م r \\
\hline 14.5 & 13.57 & 15.43 & فرات \\
\hline 12.83 & 11.73 & 13.93 & برنامج ؛ \\
\hline 20.85 & 19.83 & 21.87 & ياسمين \\
\hline \multirow[t]{2}{*}{14.65} & 13.87 & 15.43 & غدير \\
\hline & 15.39 & 17.38 & متوسط طرق الري \\
\hline للتداخل 2.52 & للري 0.57 & للتر اكيب 1.78 & $\%$ L.SD \\
\hline
\end{tabular}

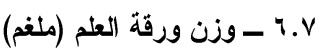

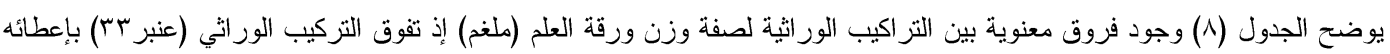

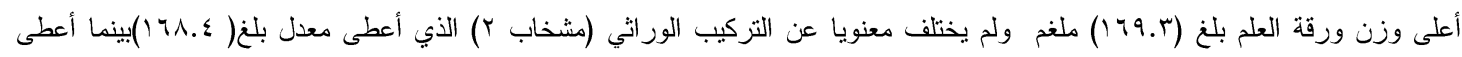

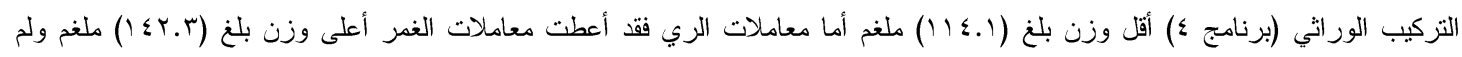

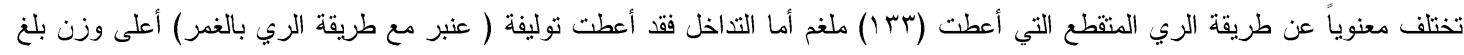

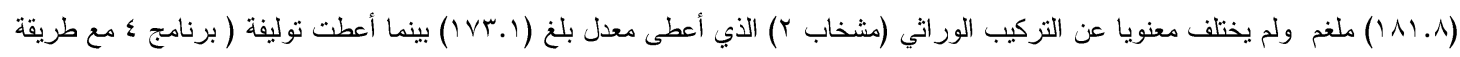

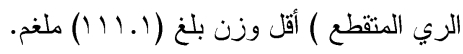

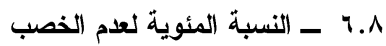

يوضح الجدول (9) وجود فروق معنوية بين التراكيب الوراثية لصفة النسبة المئوية لأخصاب إذ تثوقت التراكيب الوراثية (فرات

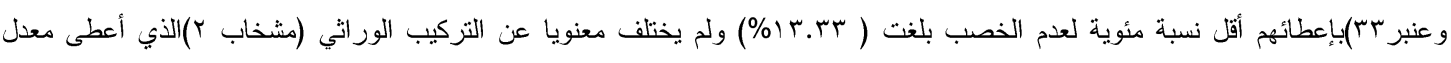

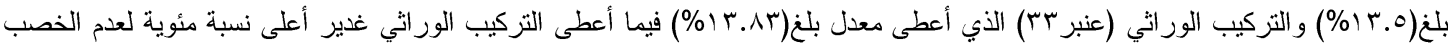

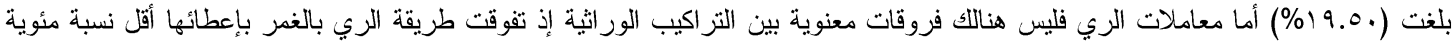

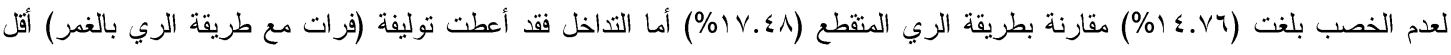

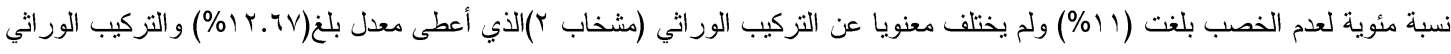

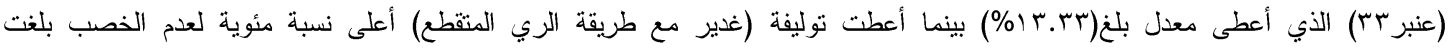
) 
جدول(^) قيم المتوسطات الحسابية لصفة وزن ورقة العلم (ملفم)

\begin{tabular}{|c|c|c|c|}
\hline متوسط التراكيب الور اثية & متقطع & غمر & 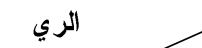 \\
\hline 169.3 & 156.8 & 181.8 & عنبر rr \\
\hline 126 & 123 & 128.9 & لدجلة \\
\hline 168.4 & 163.7 & 173.1 & مشخاب Y \\
\hline 125.5 & 122.8 & 128.3 & فرات \\
\hline 114.1 & 111.1 & 117.1 & برنامج ؛ \\
\hline 135.1 & 131.3 & 138.9 & ياسمين \\
\hline \multirow[t]{2}{*}{125.2} & 122.1 & 128.3 & غدير \\
\hline & 133 & 142.3 & متوسط طرق الري \\
\hline للتداخل 12.2 & n.s للاري & للتر اكيب 8.62 & $\%$ L.SD \\
\hline
\end{tabular}

جدول (9) قيم المتوسطات الحسابية لصفة النسبة المئوية لعدم الخصب

\begin{tabular}{|c|c|c|c|}
\hline متوسط التراكيب الور اثية & متقطع & غمر & الري ل \\
\hline 13.33 & 14.33 & 12.33 & عنبر rr \\
\hline 17.67 & 18.67 & 16.67 & لدجلة \\
\hline 13.5 & 14.33 & 12.67 & مشخاب r \\
\hline 13.33 & 15.67 & 11 & فرات \\
\hline 19.17 & 19.67 & 18.67 & برنام-ج ؛ \\
\hline 16.33 & 17.33 & 15.33 & ياسمين \\
\hline \multirow[t]{2}{*}{19.50} & 22.33 & 16.67 & غدير \\
\hline & 17.48 & 14.76 & متوسط طرق الري \\
\hline للتداخل 2.64 & للري 5.54 & للتر اكيب 1.87 & $\%$ L.SD \\
\hline
\end{tabular}

$$
\text { ( } 9.9
$$

يوضح الجدول (• () وجود فروق معنوية بين التراكيب الور اثثة لصفة عدد التقرعات الفعالة (مب) إذ تفوق التركيب الور اثي فرات بإعطائه

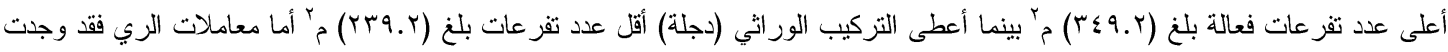

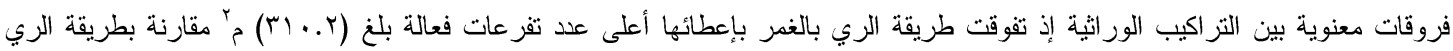

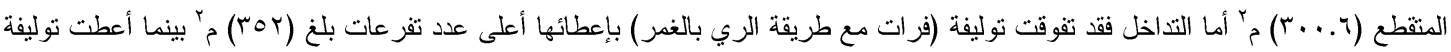

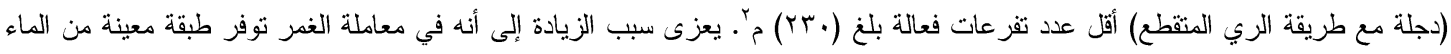

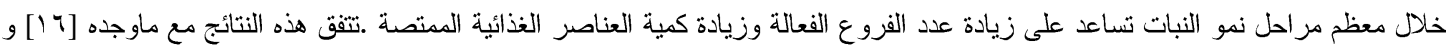


جدول (• (1) قيم المتوسطات الحسابية لصفة عدد التقرعات الفعالة (عدد الداليات.م)

\begin{tabular}{|c|c|c|c|}
\hline متوسط التر اكيب الور اثية & متقطع & غمر & الري \\
\hline 332.5 & 326.7 & 338.3 & عنبر rr \\
\hline 239.2 & 230.0 & 248.3 & لرجلة \\
\hline 332.0 & 329.0 & 335.0 & مشخاب r \\
\hline 349.2 & 346.3 & 352.0 & فرات \\
\hline 278.8 & 277.7 & 280 & برنامج ع \\
\hline 330.2 & 323.3 & 337 & ياسمين \\
\hline \multirow[t]{2}{*}{276.2} & 271.3 & 281.0 & غدير \\
\hline & 300.6 & 310.2 & متوسط طرق الري \\
\hline للتداخل 10.09 & للري 10.18 & للتر اكيب 13.50 & $\%$ L.SD \\
\hline
\end{tabular}

- V

\section{التباينات الوراثية والبيئية و المظهرية ومعاملي الاختلاف المظهري والور اثي ونسبة التوريث.}

يوضح جدول (1) (1) قيم التباينات الور اثية والبيئة المظهرية للصفات المدروسة بطريقتي الري اذ كان التباين الوراثي لصفة عن الايام من

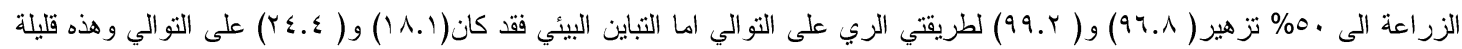

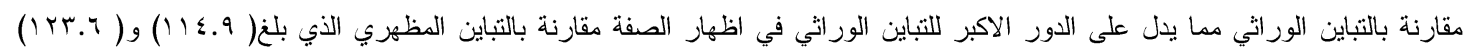

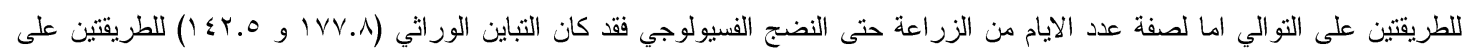

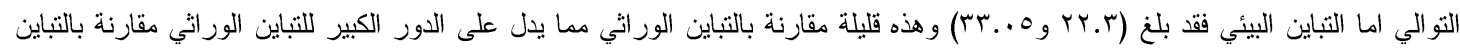

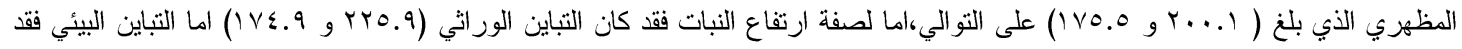

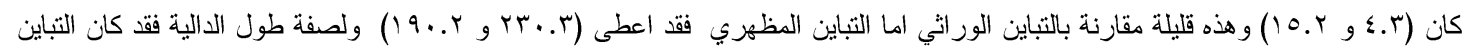

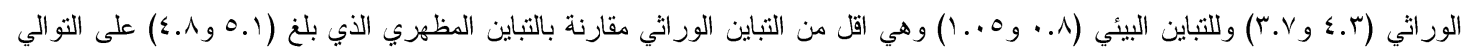

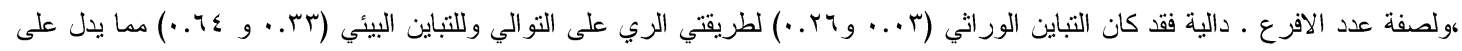

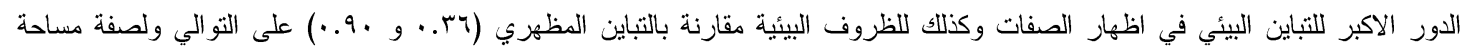

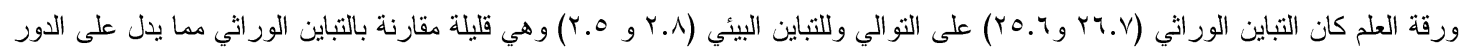

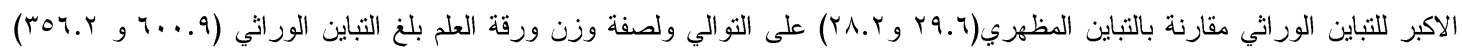

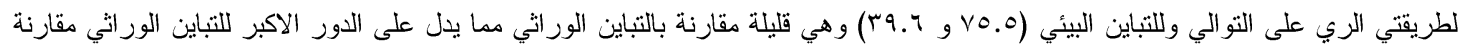

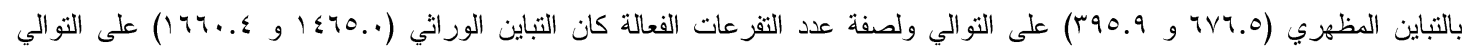

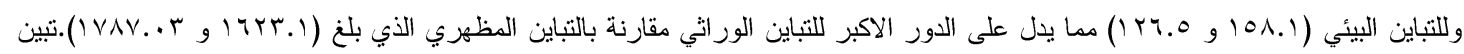

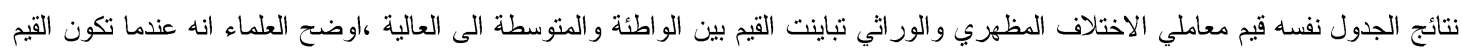

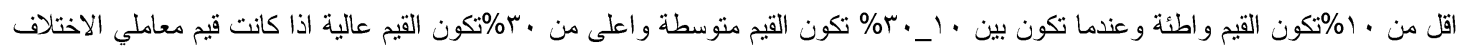

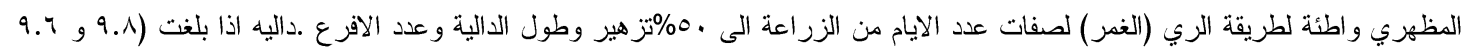

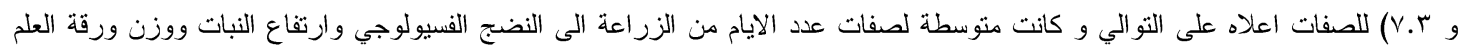

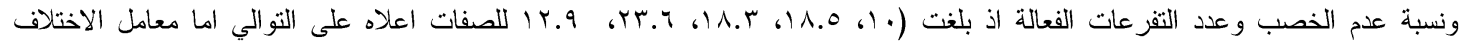

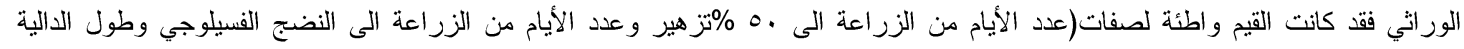

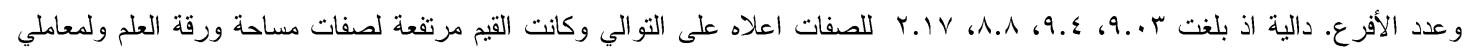

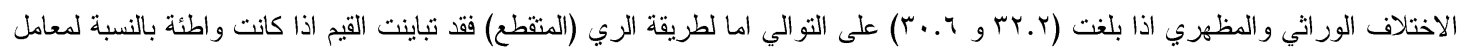

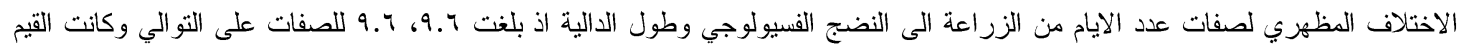

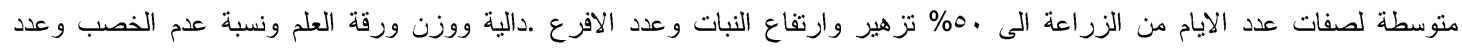




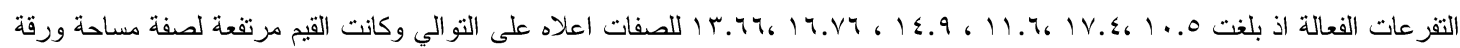

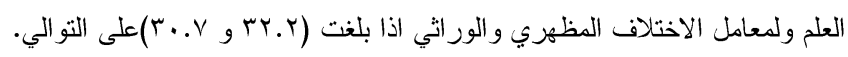

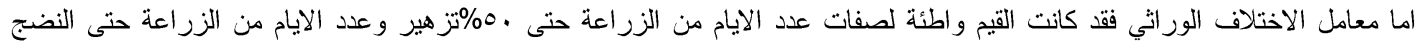

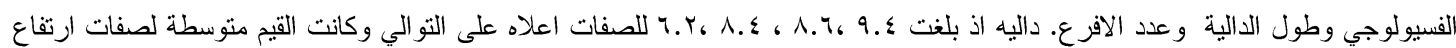

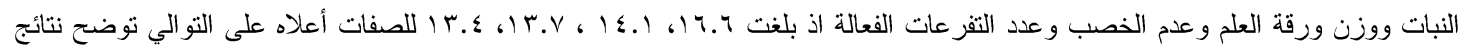

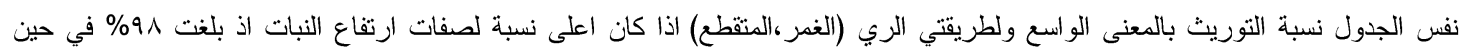

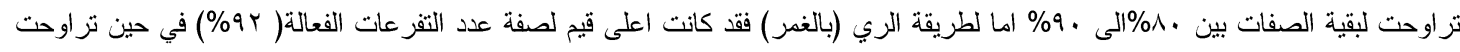

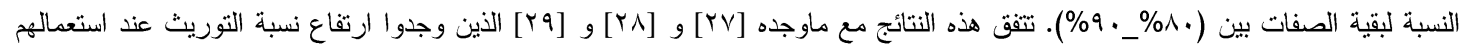

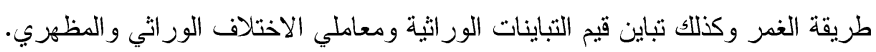




\begin{tabular}{|c|c|c|c|c|c|c|c|c|c|c|}
\hline 永 & $\begin{array}{l}\text { components of } \\
\text { variance }\end{array}$ & 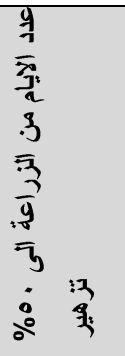 & 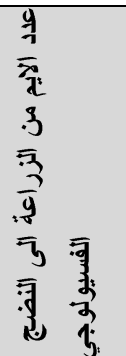 & $\begin{array}{l}\overline{\text { aj }} \\
\text { 可. }\end{array}$ & 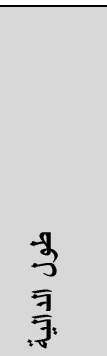 & $\begin{array}{l}\text { 季 } \\
\overline{\bar{\lambda}} \\
\mathbf{3} \\
\overline{7} \\
\overline{7}\end{array}$ & 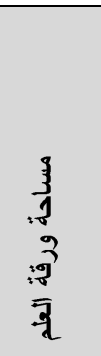 & 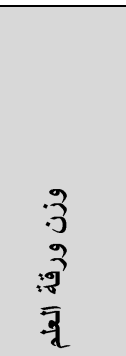 & $\frac{y}{2}$ & 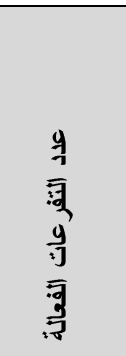 \\
\hline \multirow{6}{*}{ 霖 } & $\sigma 2 G$ & 96.841 & 177.810 & 225.944 & 4.317 & 0.032 & 26.746 & 600.992 & 10.175 & 1465.000 \\
\hline & $\sigma 2 \mathrm{E}$ & 18.111 & 22.349 & 4.357 & 0.810 & 0.333 & 2.857 & 75.548 & 2.556 & 158.190 \\
\hline & $\sigma 2 P$ & 114.952 & 200.159 & 230.302 & 5.127 & 0.365 & 29.603 & 676.540 & 12.730 & 1623.190 \\
\hline & P.C.V & 9.845 & 10.034 & 18.528 & 9.645 & 7.377 & 32.276 & 18.348 & 23.636 & 12.986 \\
\hline & G.C.V & 9.036 & 9.457 & 18.352 & 8.851 & 2.175 & 30.679 & 17.293 & 21.131 & 12.337 \\
\hline & $\begin{array}{c}\text { Heritability (broad } \\
\text { sense)\% }\end{array}$ & 84.245 & 88.834 & 98.108 & 84.211 & 8.696 & 90.349 & 88.833 & 79.925 & 90.254 \\
\hline \multirow{6}{*}{$\begin{array}{l}\bar{y} \\
\bar{y}: \\
\text { 㝵 }\end{array}$} & $\sigma 2 \mathrm{G}$ & 99.222 & 142.500 & 174.929 & 3.754 & 0.262 & 25.667 & 356.278 & 5.683 & 1660.452 \\
\hline & $\sigma 2 \mathrm{E}$ & 24.413 & 33.056 & 15.294 & 1.056 & 0.643 & 2.540 & 39.690 & 2.730 & 126.579 \\
\hline & $\sigma 2 P$ & 123.635 & 175.556 & 190.222 & 4.810 & 0.905 & 28.206 & 395.968 & 8.413 & 1787.032 \\
\hline & P.C.V & 10.599 & 9.601 & 17.406 & 9.615 & 11.681 & 32.234 & 14.914 & 16.733 & 13.954 \\
\hline & G.C.V & 9.495 & 8.650 & 16.692 & 8.494 & 6.285 & 30.749 & 14.146 & 13.753 & 13.451 \\
\hline & $\begin{array}{c}\text { Heritability (broad } \\
\text { sense) \% }\end{array}$ & 80.254 & 81.171 & 91.960 & 78.053 & 28.947 & 90.996 & 89.976 & 67.547 & 92.917 \\
\hline
\end{tabular}




\section{CONFLICT OF INTERESTS}

There are no conflicts of interest.

1- Glover, D. The system of rice intensification: Time for an empirical turn. NJAS-Wagening. J. Life Sci. 57,217224. 2011.

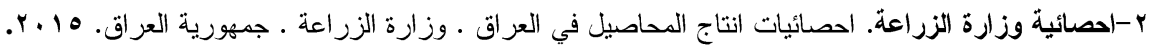

3-Bouman, B. A. Conceptual framework for the improvement of crop water productivity at different spatial scales. Agric. Syst.93, 43-60. 2007.

4- Nyamai, M.; Mati, B.; Home, P.; Odongo, B.; Wanjogu, R.; Thuranira, E. Improving land and water productivity in basin rice cultivation in Kenya through system of rice intensification (SRI). Agric. Eng.Int. CIGR J. 14, 1-9. 2012.

5- Stoop, W.A.; Adam, A.; Kassam, A. Comparing rice production systems: A challenge for agronomic research and for the dissemination of knowledge-intensive farming practices. Agric. Water Manag. 96, 1491-1501. 2009.

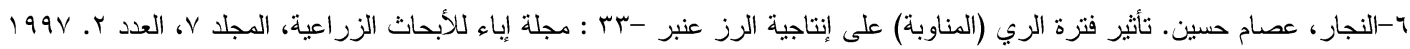

7-FAO, The State of the World's Land and Water Resourcesfor Food and Agriculture. Managing systemsat risk. 2011.

8-Chenu K. Characterizing the crop environment-nature,significance and applications. In: Sadras VO, Calderini DF, eds. Cropphysiology. Applications for Genetic Improvement and Agronomy.Burlington. MA: Academic Press, 321-348. 2014.

9-Rhine, M. D., G. Stevens, J. W. Heiser, and E. Vories. Nitrogen fertilization on center pivot sprinkler irrigated rice.Online. Crop Management doi:10.1094/CM-2011-1021-01-RS. 2011.

10-Palaniswamy, K. M. and K. A. Gomez , "Length - width method for estimating leaf area of rice", Agron J. , 66 ; 430-433. 1971 .

11-Steel,R. G. D. and J. H. Torrie, "Principles and procedures of statistics", McGraw, Hill Book Company. INC. U.S.A. 1960.

12-Walter. S.(1975) Manual of Quantitative Genetics( $3^{\text {rd }}$ edition) Washington State Univ. Press. U.S.A, Sited by A $1 \mathrm{Ha} 7 \mathrm{aa}(2001)$.

13-Hanson, C.H, H.F. Roubuson and Comstock. Biometerical studies of yield in seger gating population of Kovean Lespedeza. Agron.J. 48:268-272. 1956

14-Falconer. D. S. Interduction To Quantitative Genetics longman Group. Limited. London. 1985.

15- Thomson MJ, Edwards JD, Septiningsih EM, Harrington SE and McCouch SR,. Substitution mapping of dth1.1, a flowering-time quantitative trait locus (QTL) associated with transgressive variation in rice, reveals multiple sub-QTL. Genetics 172:2501-2514. 2006.

16- Thakur, A.K.; Rath, S.; Patil, D.; Kumar, A. Effects on rice plant morphology and physiology of water and associated management practices of the system of rice intensification and their implications for crop performance. Paddy Water Environ. 9, 13-24. 2011.

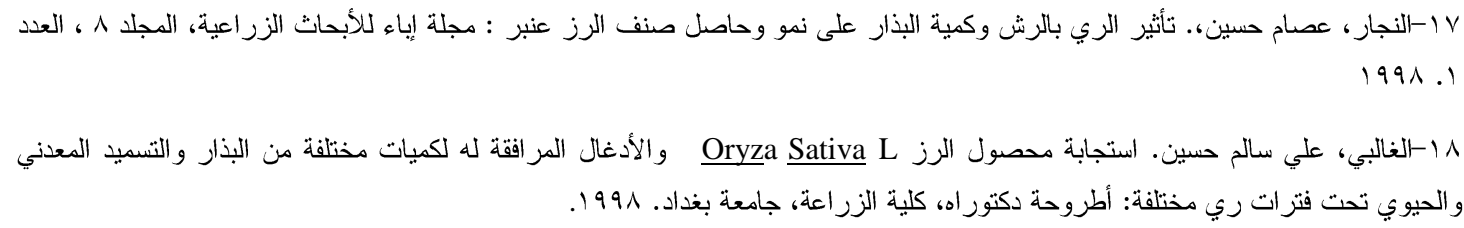

19-Zhang, H.; Xue, Y.; Wang, Z.; Yang, J.; Zhang, J. An alternate wetting and moderate soil drying regime improves root and shoot growth in rice. Crop Sci. 49, 2246-2260. 2009.

20-Chang, Y.-C.; Uphoff, N.T.; Yamaji, E..A conceptual framework for eco-friendly paddy farming in Taiwan, based on experimentation with system of rice intensification (SRI) methodology. Paddy Water Environ. 14, 169183. 2016.

21-Bernier, J.; Atlin, G. N.; Serraj, R.; Kumar, A.; Spaner, D. Breeding upland rice for drought resistance. Journal of the Science of Food and Agriculture, v.88, p.927-939. 2008.

22- Lin, X.; Zhu, D.; Lin, X. Effects of water management and organic fertilization with SRI crop practices on hybrid rice performance and rhizosphere dynamics. Paddy Water Environ. 9, 33-39. 2011. 
23- Tan, X.; Shao, D.; Liu, H.; Yang, F.; Xiao, C.; Yang, H. Effects of alternate wetting and drying irrigation on percolation and nitrogen leaching in paddy fields. Paddy Water Environ. 11, 381-395. 2013.

24-. Mishra, A.; Salokhe, V. Flooding stress: The effects of planting pattern and water regime on root morphology, physiology and grain yield of rice. J. Agron. Crop Sci. 196, 368-378. 43. 2010.

25- Kima, A.S.; Chung,W.G.;Wang, Y.-M. Improving irrigated lowland rice water use efficiency under saturated soil culture for adoption in tropical climate conditions. Water 6, 2830-2846. 2014

26-Chapman SC. Use of crop models to understand genotype by environment interactions for drought in real-word and simulated plant breeding trials. Euphytica 161, 195-208. 2008.

27- Steele KA, Price AH, Shashidar HE and Witcombe JR. Marker-assisted selection to introgress rice QTLs controlling root traits into an Indian upland rice variety. Theoretical andApplied Genetics 112:208-221. 2006.

^ץ-ايوب ،محمد حامد. الارتباط وتحليل معامل المسار وادلة الانتخاب لحاصل الحبوب ومكوناته في حنطة الخبز .مجلة علوم الر افدين، مجلد r... $\{(1)) \mathrm{V}$

29-Serraj, R.; Kumar, A.; Mcnally, K. L; Slamet-Loedin, I.; Bruskiewich, R.; Mauleon, R.; Cairns, J.; Hijmans, R. J. Improvement of drought resistance in rice. Advances in Agronomy, v.103, p.41-99. 2009. 